\title{
EDITORIAL
}

\section{SOBRE LOS ESTUDIOS LONGITUDINALES EN EPIDEMIOLOGÍA}

\author{
Cristina Pérez Andrés (1) y José María Martín Moreno (1,2) \\ (1) Comité de Redacción de la Revista Española de Salud Pública. Dirección General de Salud Pública. Ministerio \\ de Sanidad y Consumo. \\ (2) Director General de Salud Pública. Ministerio de Sanidad y Consumo. Madrid.
}

En este editorial presentamos el segundo número monográfico elaborado mediante contribuciones de los lectores/colaboradores de la Revista Española de Salud Pública a partir de una aproximación metodológica específica. Los motivos para que en esta ocasión nos hayamos centrado en los estudios longitudinales, tienen que ver tanto con la necesidad de que los resultados de los mismos se publiquen en revistas que se editan en español, como por el hecho detectado por el comité de redacción de la revista, a partir de los trabajos que recibe, relativo a la aparente confusión que existe entre algunos autores sobre lo que es un estudio longitudinal. En determinados medios no especializados se piensa que puede hablarse de un estudio longitudinal cuando el período de tiempo que se tarda en recoger los datos para la investigación, bien sea por encuesta o utilizando registros, es suficientemente dilatado. Lo cierto es que un estudio longitudinal implica el seguimiento sistematizado de variables de exposición y efecto, a partir de la observación de cómo se modifican en las mismas personas dichas variables en el tiempo. En los estudios longitudinales es posible verificar que la exposición antecede a la aparición del evento tipificado como efecto. Dado que las conclusiones que se pueden extraer con los estudios longitudinales pueden llegar a inferir la causalidad de una determinada condición, ya sea de salud o de enfermedad, así como la pro- pia historia natural de la enfermedad ${ }^{1}$, es evidente que resulta inadecuado y puede llegar a inducir grave error hacer este tipo de asociaciones a partir de estudios que carecen del diseño necesario para llegar a fundamentar estas inferencias ${ }^{2}$. Por ello pensamos que mostrando la teoría y la práctica de los estudios longitudinales, los lectores de la revista pueden llegar a constatar las diferencias que hay entre los estudios longitudinales y los que no lo son, incluyendo la diferencia entre los recursos necesarios para abordar estos estudios frente a los propios que se requieren para los estudios transversales.

Sin entrar en las disyuntivas terminológicas o conceptuales sobre el tema, que quedan bien discutidas en los artículos que componen este monográfico y que después describiremos de forma sintética, el modelo conceptual de los diseños longitudinales puede presentarse bien en estudios observacionales o bien en estudios de intervención. En el escenario de estudios de observación, podemos llegar a abordar la longitudinalidad en el diseño de forma prospectiva o retrospectiva $^{3}$. En cualquier caso, cuando hablamos de estudios longitudinales estamos invocando la idea de seguimiento de variables individuales, de medidas evolutivas (o al menos de «antes y después» en un perio- 
do), y del análisis apropiado para tener en cuenta lo anterior ${ }^{4}$

El principal recurso utilizado en los estudios longitudinales es el tiempo. Los efectos de las diferentes condiciones que se dan durante la vida de los seres humanos sólo pueden asociarse con sus causas (léase alimentación, hábito de fumar, consumo de diferentes sustancias...) si dicha asociación se formula a partir de datos generados tras emplear suficiente tiempo de observación (al menos equivalente al denominado periodo de inducción necesario para que una exposición llegue a generar biológicamente un efecto sobre la condición que se está examinando $)^{5}$. Además, durante dicho periodo deben hacerse diferentes mediciones en distintos intervalos (al menos para caracterizar el «antes» y el «después») con la condición, absolutamente necesaria, de que las personas observadas sean las mismas.

Lo que se mide en un estudio longitudinal son los potenciales cambios (el movimiento) de una variable en función del tiempo que los determinantes de dicho cambio han estado actuando. Por ello en los cálculos utilizados una de las variables que ha de estar presente, al menos de forma subyacente, es el tiempo.

El tiempo, como recurso, influye en un estudio longitudinal a través de diferentes aspectos. No sólo interviene la duración propia del estudio, en función del lapso que conlleva la aparición de la condición estudiada., sino también a través del tiempo que las personas participantes permanecen en el estudio, el cual pueden abandonar por diferentes causas, como son la aparición de la condición estudiada, un cambio de domicilio, su decisión de no seguir participando, por fallecimiento u otras. También influye el tiempo que los investigadores y personas que realizan las mediciones permanecen en el estudio sin ser sustituidos por otros (sustitución que podría introducir sesgos de observación). El avance en el tiempo de las técnicas de medición y los nuevos conocimientos aportados por otros estudios sobre las mismas condiciones estudiadas, también pueden interferir en el desarrollo de un estudio longitudinal al condicionar sus criterios de diseño y metodología. Todo lo anterior se manifiesta de forma más intensa lógicamente en los diseños tipificados como de cohorte prospecti$v a^{6}$, aunque los conceptos subyacentes de tiempo e inferencia forman parte consustancial de todos los estudios longitudinales.

Somos seres temporales no sólo porque somos mortales. Durante el tiempo que dura la vida de cada persona se producen cambios cualitativos en su organismo, relacionados con factores unas veces genéticos y otras ambientales, en cualquier caso ambos sometidos al transcurso del tiempo. Aristóteles decía en su Física que el tiempo es movimiento, cambio, y lo definía como la medida del movimiento según el antes y el después ${ }^{7}$. Posteriormente, los estoicos introdujeron las nociones de intervalo y velocidad ${ }^{7}$. A partir de Kant el tiempo pasó a ser una noción que constituye uno de los problemas constantes de la reflexión filosófica y científica (como objeto de estudio en sí mismo). Para Kant el tiempo es la forma trascendental por excelencia en la constitución de la objetividad del conocer $^{7-9}$. Todas estas nociones filosóficas sobre el tiempo no son ajenas a la ciencia en general ni a los estudios epidemiológicos longitudinales en particular. Así, en los estudios de cohortes medimos cambios o movimientos de una variable, durante intervalos definidos, y tratando de identificar a qué velocidad se produce el cambio o la transición entre condiciones de salud.

Reiterando que el tiempo es el principal protagonista en los estudios longitudinales, 
permítasenos ahora describir de forma resumida las contribuciones a este tema que hacen posible este monográfico.

El primer trabajo de este número es la colaboración de Delgado y Llorca, Estudios longitudinales: concepto y particularidades. Tras definir el concepto de estudio longitudinal, teniendo en cuenta lo que entienden las diferentes escuelas de epidemiología y las de estadística, examina cuestiones de interés en este campo y concluye que el análisis epidemiológico debe tener en cuenta las medidas repetidas, puesto que es precisamente esto lo que confiere finalmente a un estudio el mejor valor de su carácter longitudinal $^{10}$.

En el trabajo de Balaguer Vintró, Los estudios longitudinales en la prevención de las enfermedades cardiovasculares, podemos leer en su introducción los antecedentes y evolución de este tipo de estudios ${ }^{11}$. En él se revisan los principales estudios longitudinales que sobre enfermedades cardiovasculares se han realizado desde 1947 en Estados Unidos, Europa y Japón hasta llegar al Proyecto MONICA de la Organización Mundial de la Salud, realizado en 32 centros diferentes de cuatro continentes, el cual es un estudio longitudinal no de observación sino de intervención, realizándose ésta sobre los factores de riesgo coronario para examinar su influencia en la morbilidad y mortalidad coronarias $^{11}$.

En el trabajo de González y colaboradores, El estudio prospectivo europeo sobre cáncer y nutrición (EPIC), se presenta un trabajo también multicéntrico que estudia la relación de los diferentes tipos de dietas con la aparición del cáncer. Sus primeros resultados muestran un efecto protector del consumo de fibras, frutas y verduras sobre el cáncer colo-rectal, un efecto también preventivo del consumo de frutas sobre el cáncer de pulmón, y de las frutas y verduras sobre el tracto digestivo superior, mientras no se ha constatado que el alto consumo de frutas y verduras tenga efecto sobre el cáncer de próstata. Por otra parte los datos muestran una asociación entre el alto consumo de grasas saturadas y el riesgo de cáncer de mama ${ }^{12}$.

Los trabajos Aplicaciones de los modelos multiniveles al análisis de medidas repetidas en estudios longitudinales, de Zunzunegui y colaboradores, y Estimación no paramétrica de la función de supervivencia para datos con eventos recurrentes, de González y Peña, versan sobre diferentes metodologías de aplicabilidad práctica en el análisis epidemiológico de los datos procedentes de estudios longitudinales ${ }^{13,14}$.

Entre los artículos originales que se incluyen en este número, ha habido variedad en el objeto de estudio. En el trabajo titulado Volumen y tendencia de la dependencia asociada al envejecimiento en la población española, de Otero y col, el objeto de estudio es la dependencia funcional de la población anciana española, y en él se concluye que la misma aparece a edades más tardías en las sucesivas cohortes. Esto implica una mayor esperanza de vida libre de discapacidad y una mayor complejidad de los cuidados necesarios para las poblaciones dependientes, que estarán constituidas cada vez más por mujeres muy ancianas ${ }^{15}$.

En el artículo Imputación del instante de inicio de seguimiento en estudios longitudinales: aplicación a la infección por VIH, de Pérez y col. el objetivo es mostrar diversas técnicas de imputación del inicio del seguimiento y valorar su adecuación en el marco de un estudio sobre la evolución de la infección por VIH. El trabajo muestra que los instrumentos de imputación utilizados han 
resultado de utilidad para disminuir el sesgo de supervivencia en estudios observacionales ${ }^{16}$.

El trabajo de Tomàs Abadal y col, Factores de riesgo y morbi-mortalidad coronaria a los 28 años de seguimiento de una cohorte con baja incidencia de la enfermedad: el estudio de Manresa, muestra como principales resultados que los niveles elevados de colesterol y el consumo habitual de cigarrillos se asociaron independientemente con la incidencia de cardiopatía coronaria y la mortalidad coronaria ajustada por edad, presión arterial, glucemia y el índice de masa corporal. Los niveles elevados de colesterol sérico, glucemia y consumo de cigarrillos se asociaron independientemente con el riesgo de muerte por todas las causas ${ }^{17}$.

En el original de Gabriel Sánchez y col, El proyecto EPICARDIAN: un estudio de cohortes sobre enfermedades y factores de riesgo cardiovascular en ancianos españoles: consideraciones metodológicas y principales hallazgos demográficos, se concluye que la población de ancianos, de la que es representativa este estudio, presenta una elevada prevalencia de factores de riego cardiovascular, así como tasas de incidencia de cardiopatía isquémica tres veces superiores a las de la población adulta española de mediana edad. El perfil de riesgo es significativamente peor en las mujeres que en los hombres, lo que puede ser debido a la mayor mortalidad en edades más precoces en el sexo masculino ${ }^{18}$.

En el trabajo Velocidad media de ganancia de peso y estatura en niños de 2 a 10 años pertenecientes a familias del área rural del municipio de Marinilla-Antioquia, Colombia, de Álvarez y Montoya, el objetivo fue evaluar la velocidad media de crecimiento de 259 niños de 2 a 10 años, pertene- cientes a 150 familias rurales de Marinilla, Colombia, concluyendo que la velocidad media de crecimiento no logra compensar el retraso ponderal que los niños estudiados padecen $^{19}$.

Por último, se publica el trabajo Estudio longitudinal de los reingresos en pacientes con insuficiencia cardiaca en Cataluña, 1996-1999, de Caminal y colaboradores, cuyo objeto de estudio es examinar el reingreso de pacientes con insuficiencia cardiaca, identificando que las personas que padecen enfermedad pulmonar obstructiva crónica y las que padecen cardiopatía isquémica constituyen grupos prioritarios de intervención asistencial ${ }^{20}$.

Se aportan, pues, en este número de la Revista Española de salud Pública, resultados de estudios longitudinales que investigan las principales enfermedades crónicas, transmisibles (infección por VIH) o no (cardiovasculares y cáncer), que en la actualidad son las más frecuentes causas de morbimortalidad en España, así como tres estudios longitudinales realizados con sujetos en los extremos de la vida, es decir, la infancia y la senectud. Junto con los resultados del trabajo de Caminal y col. se puede concluir la importancia que para la salud pública en general, y para la prevención de la enfermedad en particular tienen los estudios que ayudan a identificar los factores que a lo largo del tiempo se relacionan con la salud y la enfermedad. Todo ello es útil a la hora de diseñar programas de intervención y de planificar servicios sanitarios y sociales que colaboren a disfrutar de mayor salud y de mejor calidad de vida incluso durante la evolución de la enfermedad.

Para terminar este editorial, no podemos dejar de decir que cuando en octubre del año 2002 la Revista Española de Salud Pública 
publicó el número monográfico sobre metodología cualitativa, ya avanzamos nuestra idea de continuar dedicando números de la revista a temas específicos, con el fin de animar a participar en ellos a los investigadores en ciencias de la salud, a través tanto de colaboraciones especiales como de trabajos originales. Ya en aquella ocasión nos congratulábamos de lo grata que había sido aquella primera experiencia, por la acogida que había tenido entre los lectores de la revista y por su respuesta. En esta segunda ocasión volvemos a expresar nuestra satisfacción y gratitud por la colaboración de las diferentes personas expertas que han colaborado en la edición de este número monográfico sobre estudios longitudinales, bien como autores de los trabajos bien como evaluadores de los mismos. Para componer este monográfico se recibieron quince trabajos, de los cuales finalmente se publican once. Por origen geográfico los trabajos han sido remitidos desde de instituciones académicas, de investigación o de servicios de salud Pública procedentes de España (Comunidad de Madrid, Comunidad Valenciana, Andalucía, Cantabria, Murcia y Asturias) y de Iberoamérica (Chile, Cuba y Colombia).

Reiteramos nuestra satisfacción por las valiosas contribuciones que contiene este número de la Revista Española de Salud Pública. Próximamente haremos la convocatoria del siguiente monográfico $\mathrm{y}$, a partir de la positiva experiencia de los dos anteriores, de antemano agradecemos el interés y generosidad de todas las personas que colaboren en su edición.

\section{BIBLIOGRAFÍA}

1. Szklo M, Nieto J. Epidemiology: Beyond the Basics. Gaithersburg, MD: Aspen Publishers; 2000.

2. Grimes DA, Schulz KF. Descriptive studies: what they can and cannot do. Lancet 2002; 359: 145-9.
3. Kelsey JL, Whittemore AS, Evans AS, Thompson WD. Methods in observational epidemiology. 2nd ed. New York: Oxford University Press; 1996.

4. Diggle P, Heagerty P, Lian KY, Zegger S. Analysis of longitudinal data. 2nd ed. Oxford: Oxford University Press; 2002.

5. Rothman KJ, Greenland S. Modern Epidemiology. 2nd ed. Filadelfia: Lippincott-Raven; 1998.

6. Grimes DA, Schulz KF. Cohort studies: matching toward outcomes. Lancet 2002; 359: 341-5.

7. Ferrater Mora J. Diccionario de filosofía abreviado. Entrada: Tiempo. Barcelona: Editorial Sudamericana; 1996. p. 350.

8. Enciclopedia Garzanti de la filosofía. Entrada: Tiempo. Barcelona: Ediciones B, SA; 1992. p. 969.

9. Kant I. Estética trascendental del tiempo. En: Crítica de la razón pura. México: Editorial Purrúa; 1991. p. 47-57.

10. Delgado M y Llorca J. Estudios longitudinales: concepto y particularidades. Rev Esp Salud Pública 2004; 78:141-8

11. Balaguer Vintró I. Los estudios longitudinales en la prevención de las enfermedades cardiovasculares. Rev Esp Salud Pública 2004; 78: 149-166.

12. González CA, Navarro C, Martínez C, Quirós JR, Dorronsoro M, Barricarte A, Tormo MJ, Agudo A, Chirlaque MD, Amiano P, Ardanaz E, Pera G, Sánchez MJ, Berenguer A. El estudio prospectivo europeo sobre cáncer y nutrición (EPIC). Rev Esp Salud Pública 2004; 78: 167-176.

13. Zunzunegui MV, García de Yébenes MJ, Forster M, Aguilar Conesa MD, Rodríguez Laso A, Otero Á. Aplicaciones de los modelos multiniveles al análisis de medidas repetidas en estudios longitudinales. Rev Esp Salud Pública 2004; 78: 177-188.

14. González Ruiz JR, Peña EA. Estimación no paramétrica de la función de supervivencia para datos con eventos recurrentes. Rev Esp Salud Pública 2004; 78: 189-199.

15. Otero Á, Zunzunegui MV, Rodríguez-Laso Á, Aguilar MD, Lázaro P. Volumen y tendencia de la dependencia asociada al envejecimiento en la población española. Rev Esp Salud Pública 2004; 78:201-213.

16. Pérez S, Ferreros I, Hurtado I, del Amo J, Hernández-Aguado I. Imputación del instante de inicio de seguimiento en estudios longitudinales: aplicación 
a la infección por VIH Rev Esp Salud Pública 2004; 78:215-27.

17. Tomàs i Abadal L, Varas Lorenzo C, Pérez I, Puig T, Balaguer Vintró I. Factores de riesgo y morbimortalidad coronaria a los 28 años de seguimiento de una cohorte con baja incidencia de la enfermedad: el estudio de Manresa. Rev Esp Salud Pública 2004; 78:229-41.

18. Gabriel Sánchez R, Novella Arribas B, Alonso Arroyo M, Vega Quiroga S, López García I, Suárez Fernández C, Muñiz García J. El proyecto EPICARDIAN: un estudio de cohortes sobre enfermedades y factores de riesgo cardiovascular en ancia- nos españoles: consideraciones metodológicas y principales hallazgos demográficos. Rev Esp Salud Pública 2004; 78:243-55.

19. Álvarez Uribe MC, Montoya Puerta EC. Velocidad media de ganancia de peso y estatura en niños de 2 a 10 años pertenecientes a familias del área rural del municipio de Marinilla-Antioquia, Colombia. Rev Esp Salud Pública 2004; 78:257-66.

20. Caminal J, Hermosilla E, Sánchez E, Urrea M, Puig $\mathrm{P}$, Tomàs Abadal L. Estudio longitudinal de los reingresos en pacientes con insuficiencia cardiaca en Cataluña, 1996-1999.Rev Esp Salud Pública 2004; 78:267-76. 